\title{
Research on Model Beginning Standard of Multi-objective Evolutionary Algorithm Based on Entropy
}

\author{
Qiong Yuan ${ }^{1,2}$, Guangming Dai ${ }^{1,2 *}$ \\ 1 School of Computer Science, China University of Geosciences, Wuhan 430074, China. \\ 2 Hubei Key Laboratory of Intelligent Geo-Information Processing, China University of Geosciences, Wuhan \\ 430074, China. \\ * Corresponding author. Tel.: 13971106905; email: yqszb@126.com \\ Manuscript submitted March 31, 2016; accepted May 15, 2016. \\ doi: 10.17706/jsw.11.7.685-694
}

\begin{abstract}
Entropy is a measurement of the system's degree of chaos, it has important applications in the field of control theory, probability theory, number theory, astrophysics and life sciences. In the process for multi-objective evolutionary algorithm to solve optimization problem, the distribution of individuals in the population goes from disorder to order. The paper introduces the entropy theory to multi-objective evolutionary and proposes an entropy calculation method to describe the population distribution, besides, it offers a method to judge the critical point, which can be used as a model beginning standard.
\end{abstract}

Key words: Multi-objective evolutionary algorithm, entropy, critical point, model beginning standard.

\section{Introduction}

In the study of hybrid multi-objective evolutionary algorithm [1]-[3], in addition to the traditional crossover and mutation operator, a probabilistic model that describes the distribution of solution set by the means of statistical learning from the macro-perspective was introduced to reproduce new individuals. As the proposal of such method is not long, its theoretical system is not so mature. Therefore, the further research on it has very important theoretical significance and innovative value. In addition, at the initial phase of algorithms, as global information is insufficient, the search with the method of probability modeling usually will make it far away from objective method. How to get the traditional multi-objective algorithm combined with the method of probabilistic model better and learn from mutual advantages deserves a deep research.

In the process of optimization problem for multi-objective evolutionary algorithm, the individual distribution in population exists with phenomenon from disorder to orderliness [4]-[9]. By introducing the entropy theory into multi-objective evolution, this paper proposes a kind of computing method about entropy to describe the distribution of population, and does experiment on ZDT test set with NSGA-II algorithm, and then introduces it into the research of multi-objective evolution as a kind of new modeling beginning standard.

\section{Entropy and Critical Point in Multi-objective Evolution}

\subsection{Entropy in Multi-objective Evolution}

Definition 1. Entropy of population is a kind of measurement of all individual distribution in solution space. If the Pop scale in evolution population is $N$ and the dimensionality of decision variable is $n$, then each dimensionality in decision space is divided into $\mathrm{K}$ sections, then the grid number of whole decision 
space is $K^{n}$, the entropy of defined population is:

$$
E=-\sum_{i=1}^{K^{n}} p_{i} \log p_{i} \quad\left(p_{i} \neq 0\right)
$$

where, $\mathrm{pi}=$ counter $[\mathrm{i}] / \mathrm{N}$, counter[i] is the number of individual in No. $\mathrm{i}$ grid.

\subsection{Critical Point in Multi-objective Evolution}

Definition 2. Critical point in multi-objective evolution. In the entropy graph of population in the evolutionary process, the point $c$ is called as the critical point of multi-objective evolution, which satisfies:

$$
\frac{\left|E_{c}-E_{c+k}\right|}{k}<\varepsilon
$$

where, $E_{c}$ is the entropy of No. c evolutionary population, and $E_{c+k}$ is the entropy of No. (c+k) population, and $\varepsilon$ is a constant.

\section{Theoretical Entropy and Numerical Experiment of Test Set}

\subsection{Theoretical Entropy of ZDT Test Set}

ZDT test set was proposed by E.Zitzler, K. Deb and L.Thiele in 2000 [10], and it presents many characters which described in Deb's Toolkit, including different front shape (such as the front of ZDT1 is convex, while the front of ZDT2 is not), discrete problem ZDT3, multiple front problem ZDT4 and preference problem ZDT6. All problems are provided with two objectives, consisting of function $\mathrm{f} 1, \mathrm{~g}$ and $\mathrm{h}$. The form is expressed as follows:

$$
\begin{aligned}
& \text { Minimize } F(x)=\left(f_{1}\left(x_{1}\right), f_{2}(x)\right) \\
& \text { subject to } f_{1}=x_{1} \\
& \qquad f_{2}=g\left(x_{2}, \cdots, x_{m}\right) h\left(f_{1}\left(x_{1}\right), g\left(x_{2}, \cdots, x_{m}\right)\right) \\
& \text { where } \quad x=\left(x_{1}, \cdots, x_{m}\right)
\end{aligned}
$$

$P F_{\text {true }}$ of ZDT1-ZDT4 and ZDT6 are all corresponding $g(x)=1$, whereas:

$$
g(x)=\left\{\begin{array}{l}
1+9 \sum_{i=2}^{m} x_{i} /(m-1) \text { for ZDT1-ZDT3 } \\
1+10(m-1)+\sum_{i=2}^{m}\left(x_{i}^{2}-10 \cos \left(4 \pi x_{i}\right)\right) \text { for ZDT4 } \\
1+9\left(\sum_{i=2}^{m} x_{i} /(m-1)\right)^{0.25} \text { for ZDT6 }
\end{array}\right.
$$

Therefore, to achieve optimal solution, the decision space includes:

$$
x_{1} \in[0,1] x_{i}=0(i=2, \cdots, m)
$$

According to the computing method of entropy listed in Formula (1), the entropy when population convergence to PF only is related to the value of decision variable in the first dimensionality. At this time, all individuals gather in the top $\mathrm{K}$ grids, and are equably distributed in these grids. The theoretical entropy of population is: 


$$
E=-\sum_{i=1}^{K} p_{i} \log p_{i}=-K \times \frac{1}{K} \log \frac{1}{K}=-\log \frac{1}{K}=\log K
$$

\subsection{Experimental Result and Analysis of Population's Entropy}

In order to verify the computing method for entropy provided in formula (1) and the analysis of (3) on the theoretical entropy of different test sets, NSGA-II [11] algorithm is applied to conduct experiment respectively on the ZDT test set. The population size N in NSGA-II is set to be 1000, and the evolutionary generation is set to be 250 . Simulated binary crossover is utilized and the crossover probability is 0.9 . The variation chooses polynomial variation and the variation probability is $1 / \mathrm{n}$. The dimensions of decision variables, and the interval numbers in each dimension, as well as the times setting of independent operation of experiment are shown in Table 1.

Table 1. Related Parameter Setting for Experiment

\begin{tabular}{|c|c|c|c|}
\hline Test Suit & Variable Num & Grid Num & Run Times \\
\hline ZDT & $5,8,10$ & 3,5 & 10 \\
\hline
\end{tabular}

For the purpose of describing the entropy changes in population evolution process more explicitly, we operates the algorithm for ten times in each certain condition and then provides the entropy graphs for those with better population convergence.
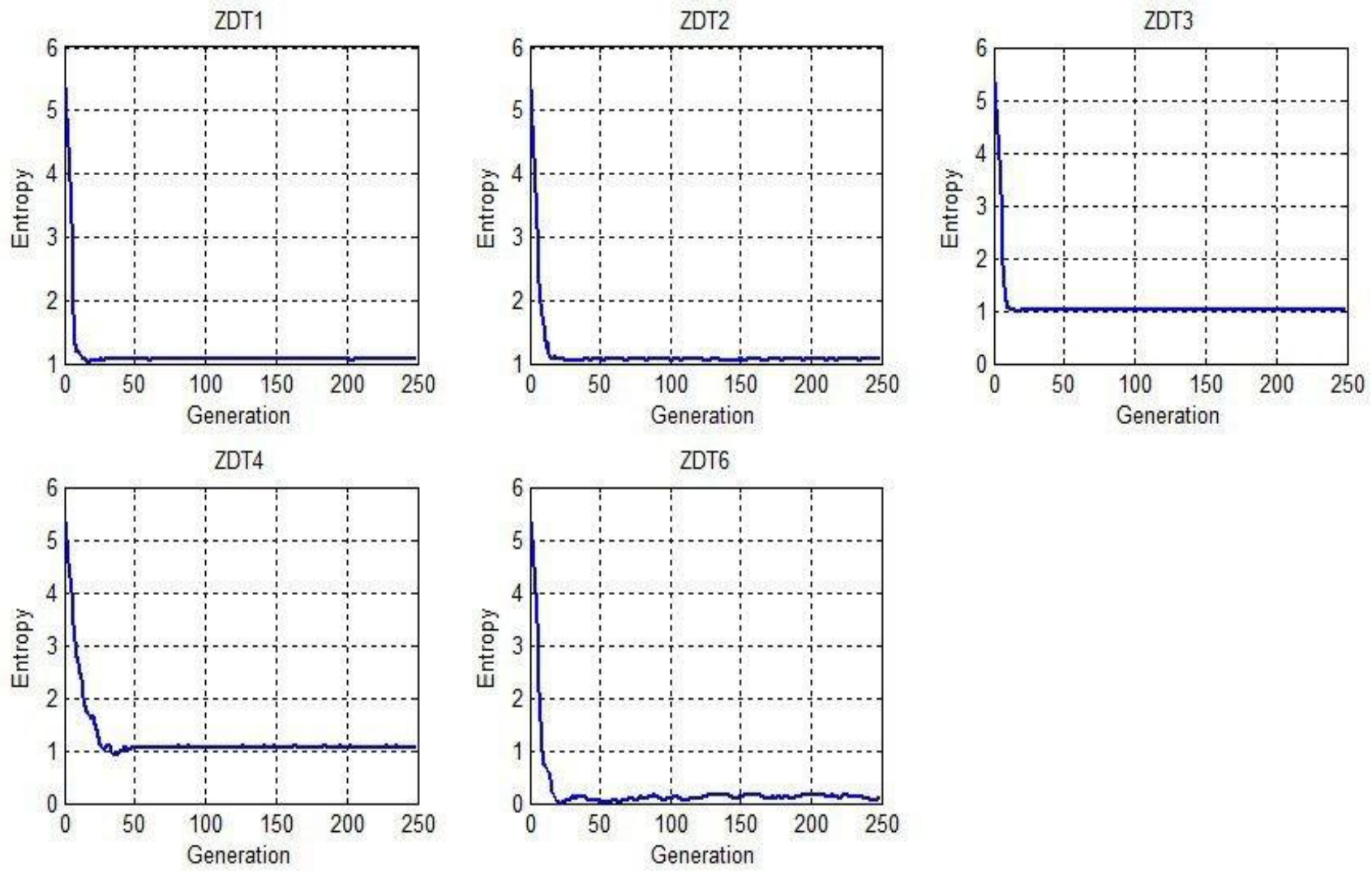

Fig. 1. Entropy graph of test set ZDT (Variable Num=5, Grid Num=3). 

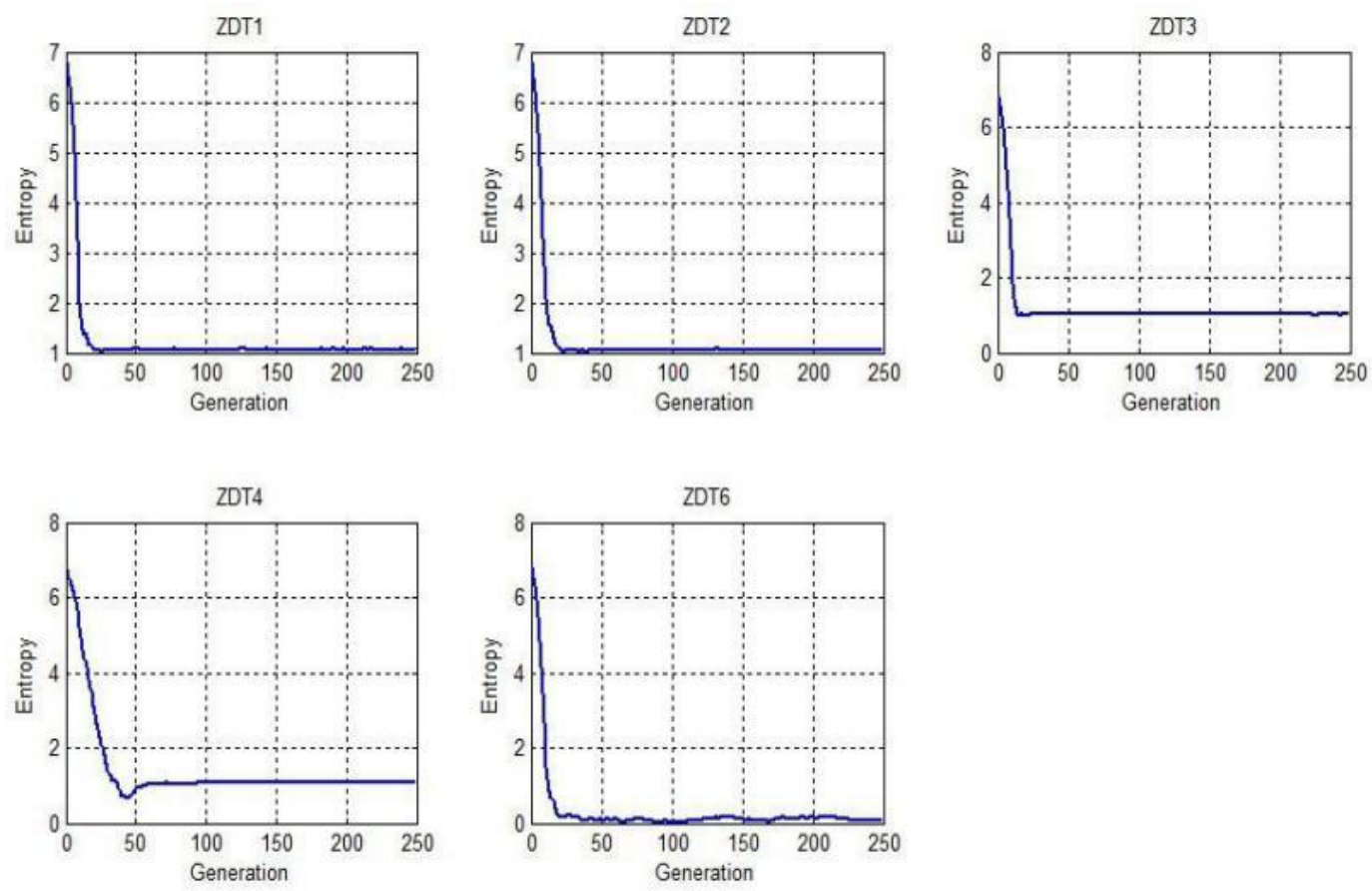

Fig. 2. Entropy graph of test set ZDT (Variable Num= 8, Grid Num=3).
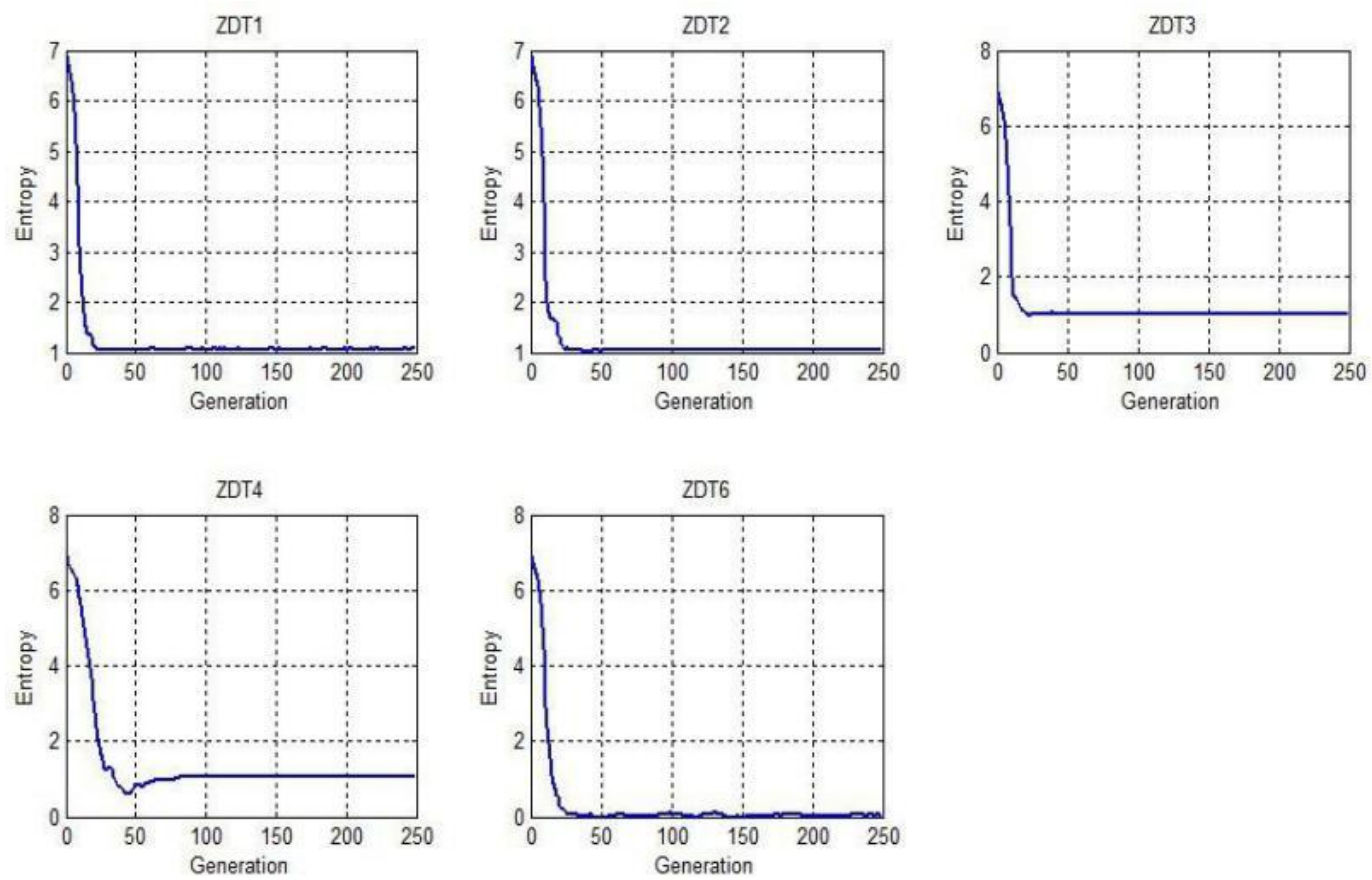

Fig. 3. Entropy graph of test set ZDT (Variable Num=10, Grid Num=3).

It can be concluded from formula (3) that when dividing three sections in each dimension of the decision space, the theoretical entropy of ZDT at the time of population convergence should be as follows:

$$
E=\log K=\log 3 \approx 1.0986
$$

Combined with the obtained entropy Fig.1 - 3, we can draw following conclusions:

1) The entropy of population is larger at early evolution, and then gradually decreases as the evolution goes on. When the evolution processes to a certain stage, the value tends to be stable.

2) As to the four test problems ZDT1-ZDT4, when the population converges or tends to be stable, the 
entropy is close to the theoretical value 1.0986. Except for ZDT6, the entropy is close to 0 when the graph tends to be stable.

3) The change of dimensions of decision spaces has no impact on the entropy when the population tends to be stable.
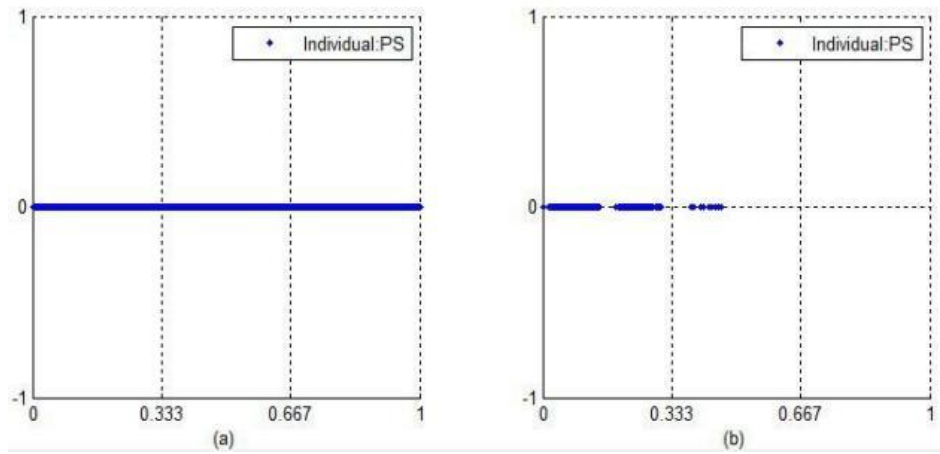

Fig. 4. Population distribution of ZDT6 in decision space.

As to the particular value of ZDT6, we conducts analysis when the number of dimensions of decision variables is 5 . Based on formula (3), the entropy at population convergence is decided by the value of individuals in the first dimension of decision space. Theoretically, individuals should be in uniform distribution among $[0,1]$. Therefore, the PS of population presents one-dimensional linear manifold as shown in Fig. 4(a). However, the PS distribution of population worked out by the algorithm is shown in Fig. 4(b) where $[0,1]$ is divided into three sections. According to the statistics, the individuals in the first section account for $98.6 \%$ and that in second section accounts for $1.4 \%$. Accordingly, the entropy of population is as follows:

$$
E=-(0.986 \times \log 0.986+0.014 \log 0.014) \approx 0.0737
$$
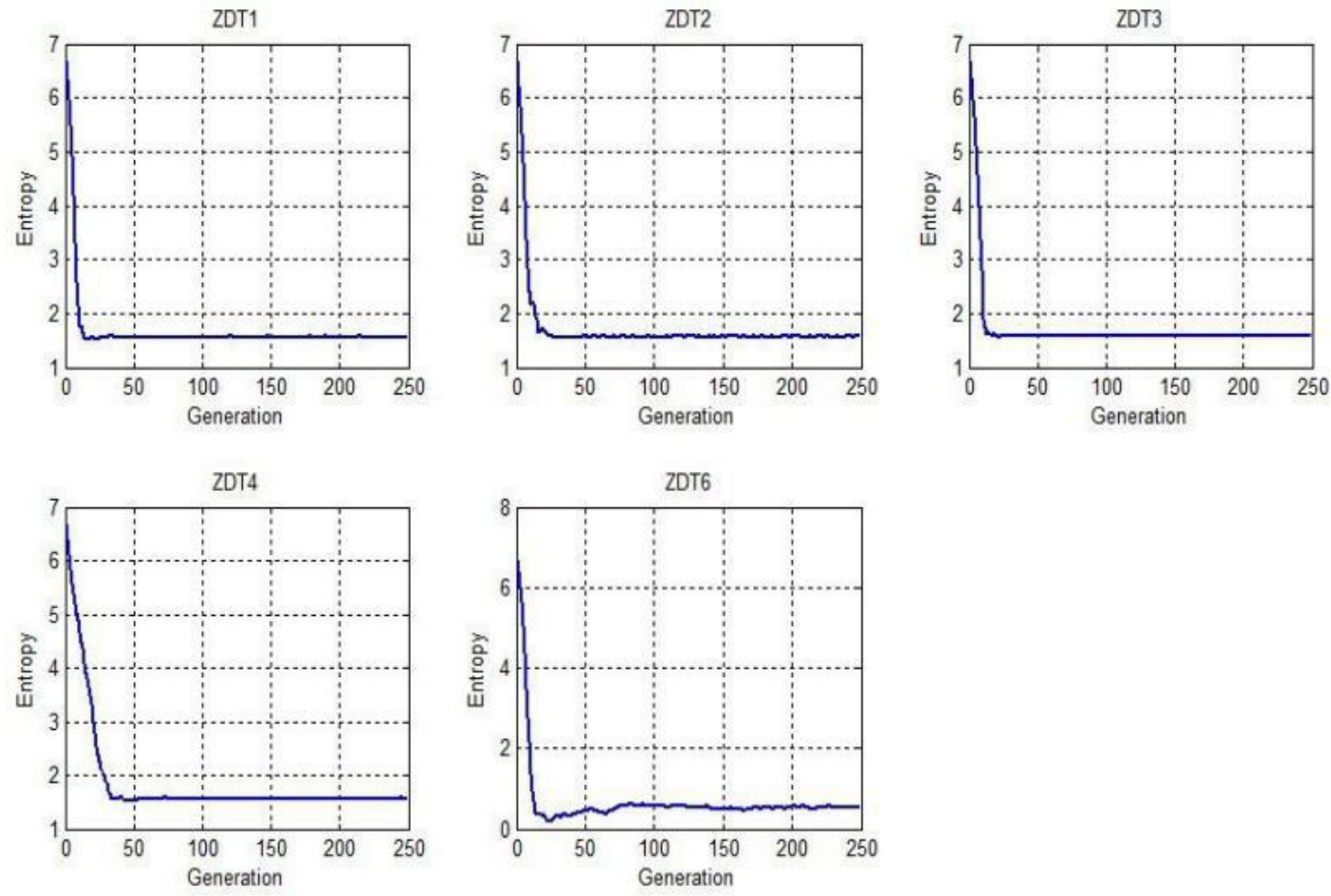

Fig. 5. Entropy graph of test set ZDT (Variable Num=5, Grid Num=5). 

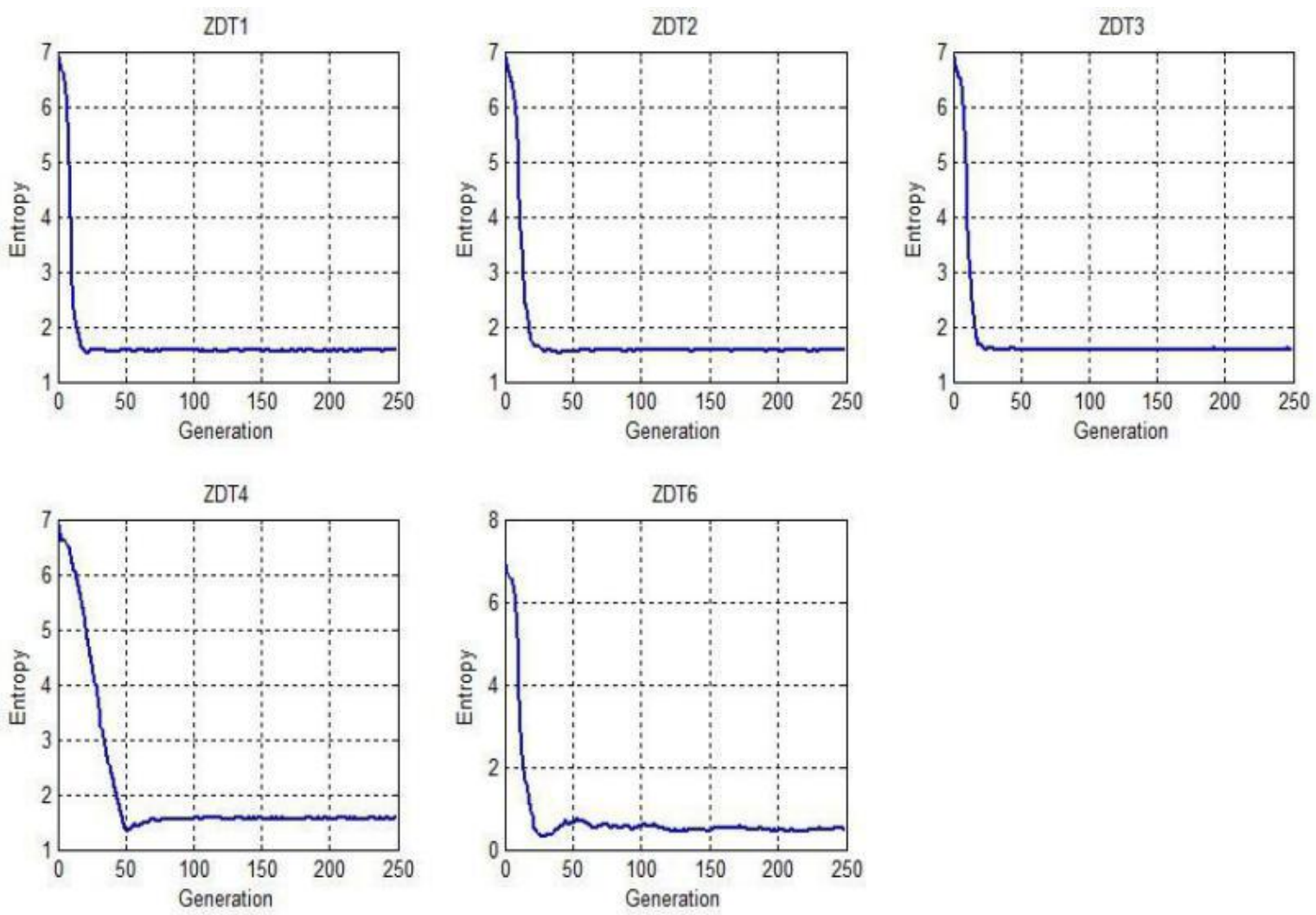

Fig. 6. Entropy graph of test set ZDT (Variable Num=8, Grid Num=5).
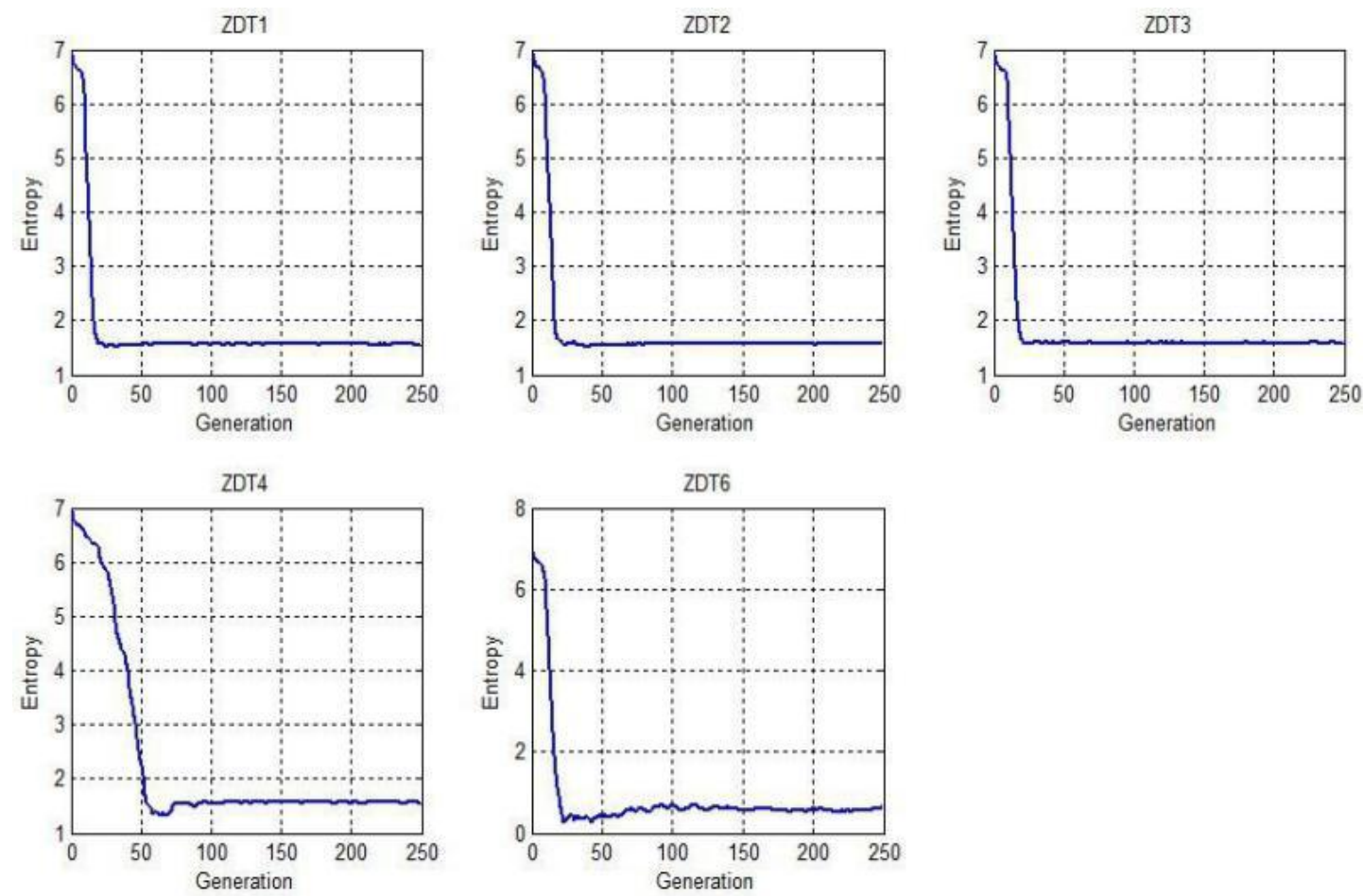

Fig. 7. Entropy graph of test set ZDT (Variable Num=10, Grid Num=5).

As to the four test problems ZDT1-ZDT4, when the population converges or tends to be stable, the entropy is close to the theoretical value 1.6094 . As to ZDT6, the solution worded out by the algorithm is nonuniform, making the entropy close to 0.5 when the graph tends to be stable. 
Comparing Fig.1-3 with Fig.5-7, the number of sections divided in each dimension of decision variables is the factor that directly impacts the entropy of population. When the number of sections divided in each dimension of decision spaces is different, the entropy at convergence turns out different, verifying the computing method for entropy defined in formula (1).

\subsection{Experimental Result and Analysis of Critical Phenomena}

When $\mathrm{k}=5, \mathrm{e}=0.1$, and based on the entropy data of test set ZDT when the number of decision variables is 5 and that of sections in each dimension is 3, formula (2) can be used to compute the critical point of multiple-object algorithm. Thus, the average rate of change of population's entropy is as shown in Fig. 8-9.
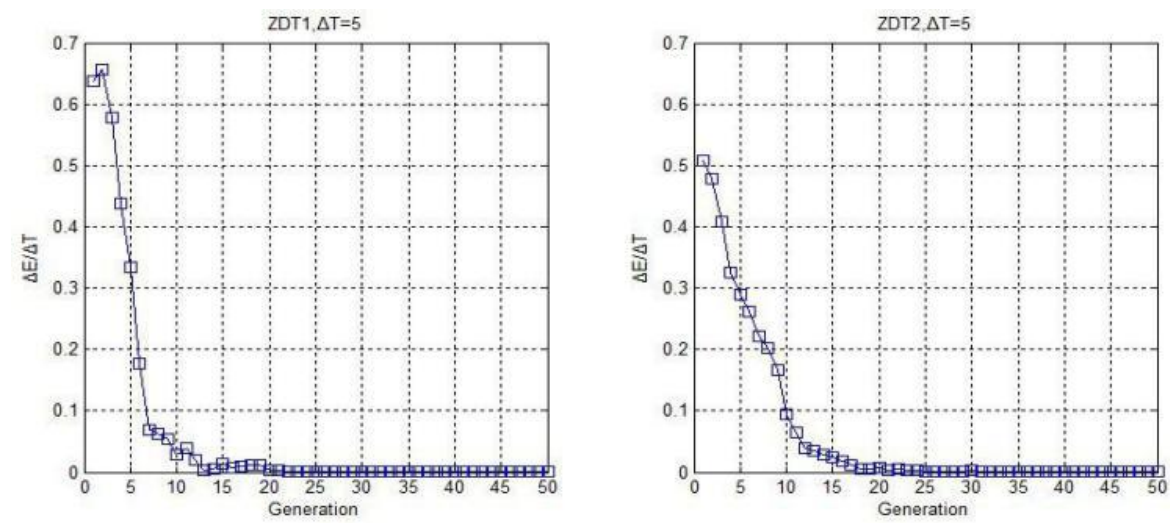

Fig. 8. Entropy difference graph of ZDT1,ZDT2.
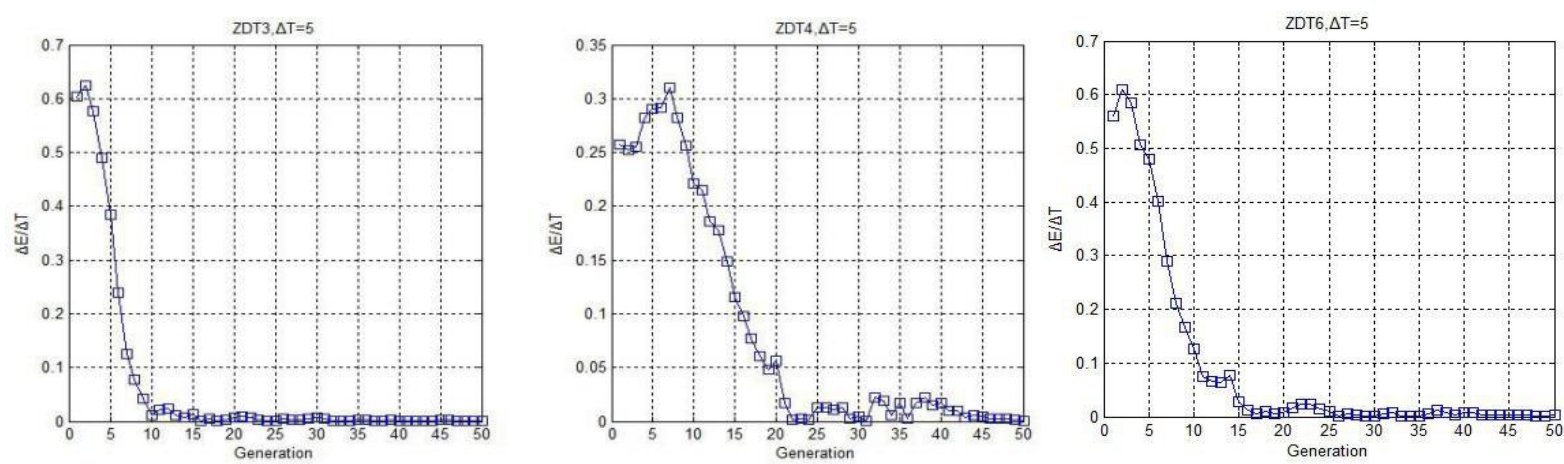

Fig. 9. Entropy difference graph of ZDT3,ZDT4,ZDT6.

1) As far as ZDT1 is concerned, the critical point that meets the conditions is 7 ( $c=7$ ), namely the five generations ranging from 7 to 11 are the critical points where the distribution transits from disorder to order. Combined with the distribution of ZDT1's solution sets in objective space as shown in Fig.10, before generation 7 , the distribution of population is provided with randomness. The population gradually converges to the shape of $P F_{\text {true }}$ and is approaching to $P F_{\text {true }}$ after the evolution from generation 7 to 11 .

2) As far as ZDT2 is concerned, the critical point that meets the conditions is 10 (c = 10). Combined with Fig.11, before generation 10, the distribution of population is provided with randomness. The population gradually converges to the shape of $P F_{\text {true }}$ and is approaching to $P F_{\text {true }}$ after the evolution from generation 10 to 14 .

3) As to ZDT3, ZDT4 and ZDT6, the critical points that meet the conditions are as shown in Table 2: 

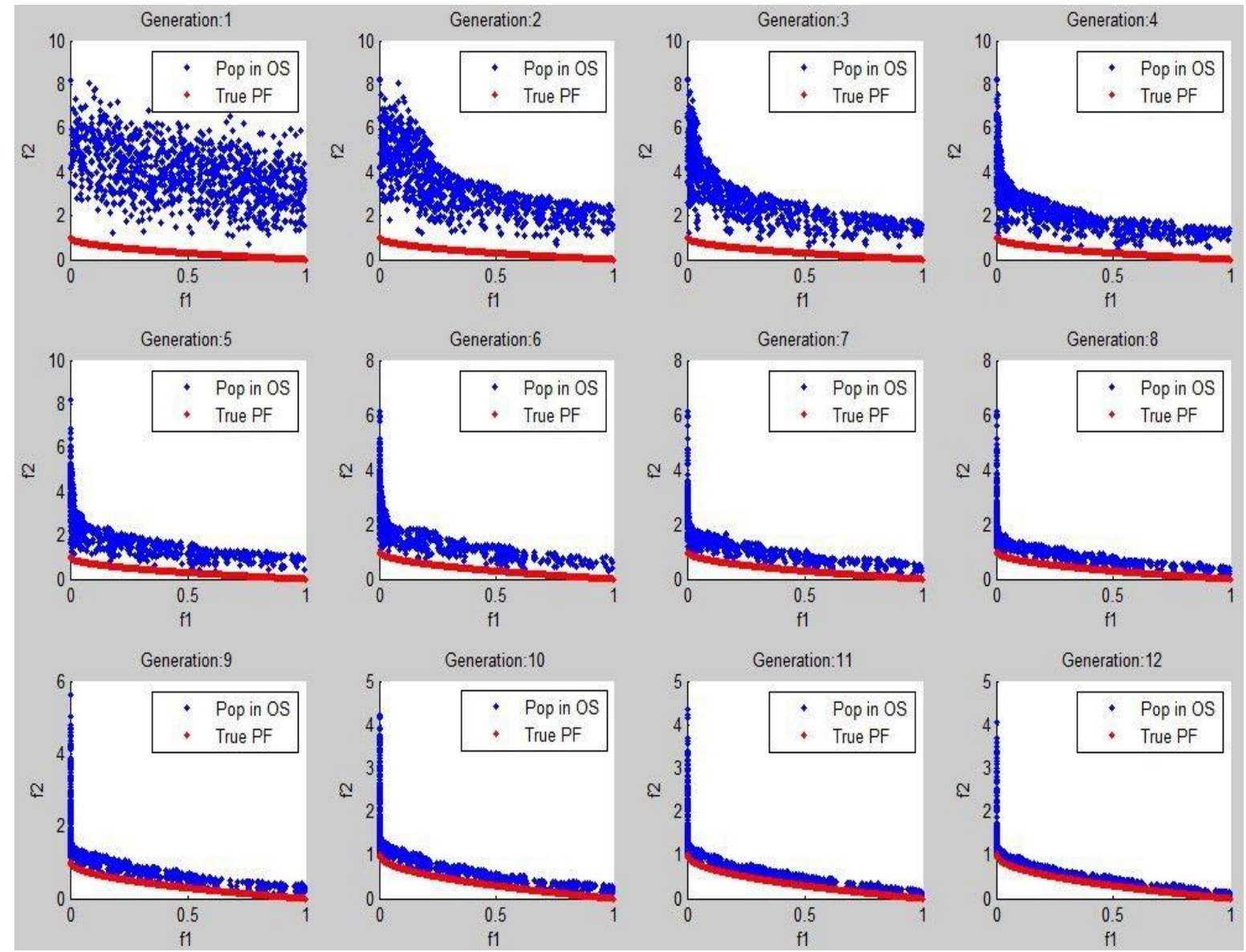

Fig. 10. Distribution of solution sets of ZDT1 in objective space.
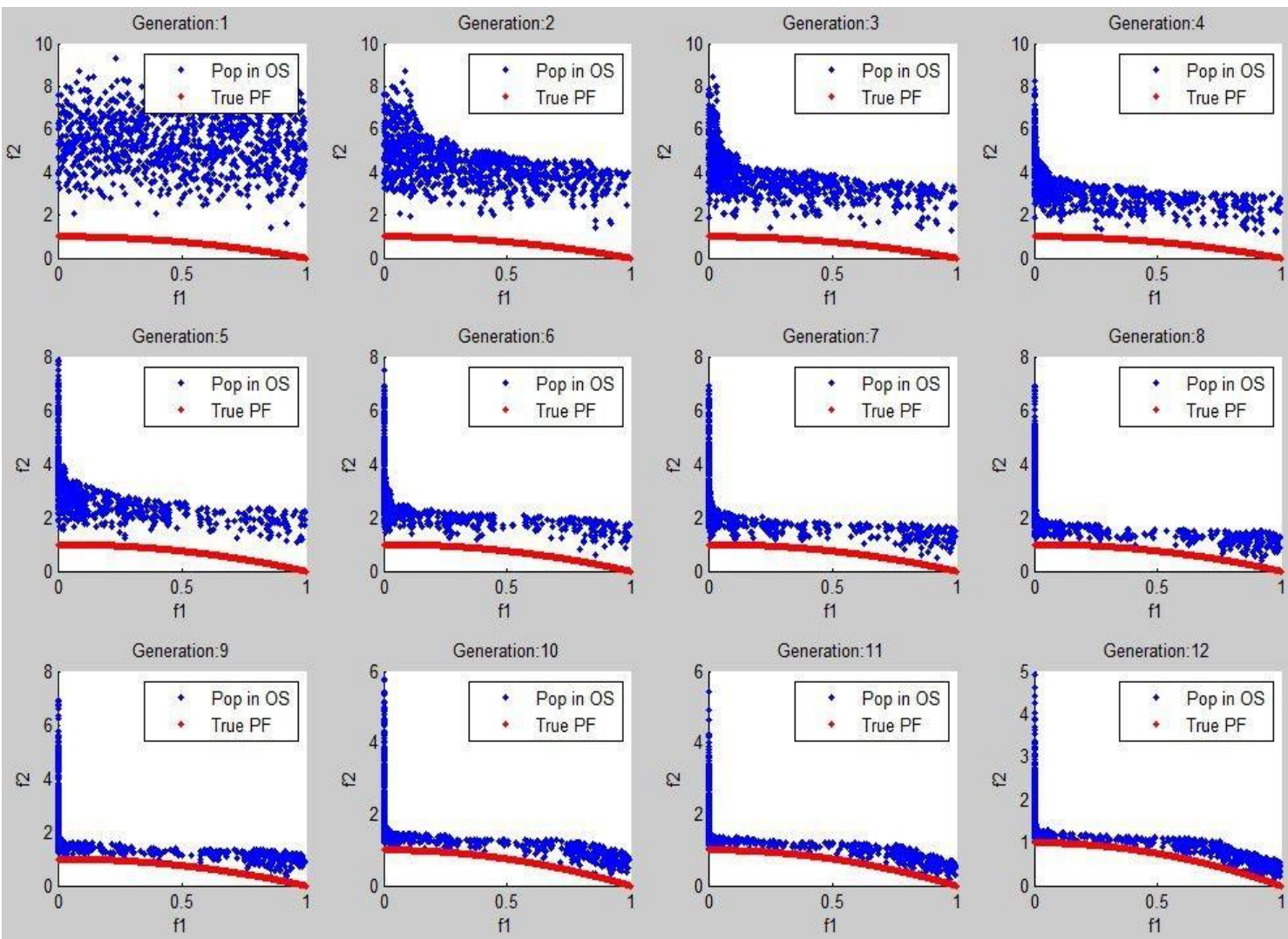

Fig. 11. Distribution of solution sets of ZDT2 in objective space. 
Table 2. The Critical Points of ZDT3, ZDT4 and ZDT6

\begin{tabular}{|c|c|c|c|}
\hline Problem & ZDT3 & ZDT4 & ZDT6 \\
\hline Critical point c & 8 & 16 & 11 \\
\hline
\end{tabular}

Combined with Fig. 8-11, before the critical point, the distribution of population is random and disordered. After the evolution for $k$ generations since critical point, the distribution of population begins to present certain regularity and approach reality. The generation $c+k$ in the evolution is the transit from disorder to order, which is a sort of critical point. The selection of $k$ and $\varepsilon$ should based on different problems.

\section{Conclusions}

The thesis introduces entropy theory into the research on multi-objective evolution and defines a computing method for entropy. Based on given computing method, the theoretical entropy of test set ZDT is analyzed when the population converges. Furthermore, NSGA-II algorithm is applied for experiment. The comparison and analysis of the entropy worked out and the theoretical value testify the correctness of the defined computing method for entropy. In addition, on the basis of entropy graph of test problem ZDT, this thesis defines the critical point in multi-objective evolutionary algorithm and conducts analysis on the critical point combined with the distribution of solution set in objective space, as well as regards the critical point as a modeling beginning standard that traditional multi-objective algorithm can be used before the critical point while the distribution estimation algorithm based on rule models should be applied after crossing the critical point, which is the work required for next stage.

\section{Acknowledgment}

This paper is supported by National Natural Science Foundation of China (No.61472375, No.61103144) and Youth Fund Project of Wuhan Donghu University (Project No. 2014) .

\section{References}

[1] Zhou, A., Zhang, Q., Jin, Y., et al. (2005). A model-based evolutionary algorithm for bi-objective. Congress on Evolutionary Computation.

[2] Zhou, A., Jin, Y., Zhang, Q., et al. (2006). Combining model-based and genetics-based offspring generation for multi-objective optimization: Using a convergence criterion. Congress on Evolutionary Computation.

[3] Zhang, Q., Zhou, A., \& Jin. Y. (2008). RM-MEDA: A regularity model-based multiobjective estimation of distribution algorithm. IEEE Transactions on Evolutionary Computation, 12(1), 41-63.

[4] Farhang-Mehr, A., \& Azarm, S. (2002). Diversity assessment of Pareto optimal solution sets: an entropy approach. Proceedings of the 2002 Congress on Evolutionary Computation, 1, 723-728.

[5] Gunawan, S., Farhang-Mehr, A., \& Azarm, S. (2003). Multi-level multi-objective genetic algorithm using entropy to preserve diversity. Evolutionary Multi-Criterion Optimization.

[6] Ocenasek, J. (2006). Entropy-based convergence measurement in discrete estimation of distribution algorithms. Towards a New Evolutionary Computation.

[7] Wang, Y. N., Wu, L. H., \& Yuan, X. F. (2010). Multi-objective self-adaptive differential evolution with elitist archive and crowding entropy-based diversity measure. Soft Computing, 14(3), 193-209.

[8] Qin, Y., Ji, J., \& Liu, C. (2012). An entropy-based multiobjective evolutionary algorithm with an enhanced elite mechanism. Applied Computational Intelligence and Soft Computing.

[9] LinLin, W., \& Yunfang, C. (2012). Diversity based on entropy: A novel evaluation criterion in multi-objective optimization algorithm. International Journal of Intelligent Systems and Applications.

[10] Zitzler, E., Deb, K., \& Thiele, L. Comparison of multiobjective evolutionary algorithms: Empirical results. 
Evolutionary Computation, 8(2), 173-195.

[11] Deb, K., Pratap, A., \& Meyarivan, T. (2002). A fast and elitist multiobjective genetic algorithm: NSGA-II. Evolutionary Computation.

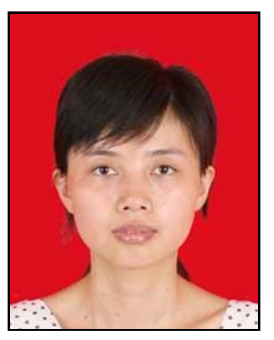

Qiong Yuan was born in Huber, China, in 1980. She received has M.S. degree in computer application technology in Huazhong University of Science and Technology in China in 2008. Since September 2013, she has been working for her PhD degree in China University of Geosciences. Her research interests are in the area of evolutionary algorithms and optimization.

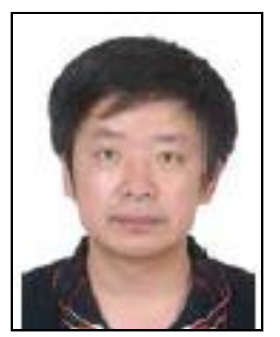

Guangming Dai was born in Anhui, China, in 1964. He received his PhD degree from Huazhong University of Science and Technology, China, in 2002. He is currently a professor in geosciences, China, since 2002. His main interests are in the area of evolutionary algorithms and algorithm design and analysis. 